\title{
La fonction argumentative des néologismes construits à partir de noms propres (sur l'exemple du sigle DSK)
}

\author{
Agnieszka Konowska \\ Institut d'Études romanes, Université de Łódź*
}

Cet article examine la créativité néologique exploitant les noms propres pour les besoins de l'argumentation discursive. En prenant pour observatoire quelques innovations construites sur le sigle propriel $D S K$, nous nous proposons de (dé) montrer que leur emploi suppose une coprésence de plusieurs fonctions différentes («fonctions-moyens ») qui sont activées ponctuellement pour servir la fonction de persuasion («fonction-fin »). Notre étude de quelques exemples ciblés de néologismes propriels (faire son DSK, être un DSK, dskiser, dskien, dskesque) met à jour les principaux mécanismes argumentatifs caractéristiques des noms propres et des créations néologiques proprielles : le recours à l'implicite et à la connivence, le fonctionnement sur le mode pathémique, le recours au bagage culturel commun constituant un passage obligé pour leur décodage.

Mots-clés : argumentation, néologie, nom propre, connivence, fonctions du langage.

\section{Introduction}

Quelques mois après l'évènement $\mathrm{DSK}^{1}$, Le Monde commentait ainsi l'apparition d'une nouvelle pratique langagière dans l'espace socio-discursif français :

« Séisme politique et médiatique, l'affaire Strauss-Kahn a aussi bouleversé les relations hommes-femmes. En particulier dans le monde de l'entreprise. Le Petit Robert devra en tirer les conséquences : "DSK" est devenu un nom commun, et "faire son DSK", une expression courante. Par prétérition, une précaution d'usage : "Je

\footnotetext{
* agnieszka.konowska@uni.lodz.pl.

${ }^{1}$ L'affaire dans laquelle Dominique Strauss-Kahn, l'ancien président du Fonds Monétaire International, a été accusé d'agression sexuelle envers une femme de chambre de l'hôtel Sofitel à New York.
} 
ne voudrais pas faire mon DSK, mais...", commence-t-on à entendre de la part des habitués de la galanterie. Un DSK est maintenant un homme qui vous saute dessus sans vous demander votre avis $»^{2}$.

L'une des fonctions fondamentales des néologismes, toujours invoquée, est de permettre de nommer les réalités nouvelles. Cependant, l'expression néologique faire son DSK désigne une pratique existant depuis des lustres et possédant déjà le nom de harcèlement sexuel. Il s'agit donc d'un néologisme nommant d'une façon nouvelle une réalité existante, mais qui jusqu'à « l'affaire DSK » restait non-dite à cause de sa tabouisation sociale. Plus que d'une fonction dénominative, il convient donc de parler ici d'une fonction euphémique du néologisme : les locuteurs ont trouvé un moyen d'évoquer publiquement une conduite dont il était difficile de parler.

L'euphémisme servant « à la construction d'un ethos favorable pour le locuteur qui laisse entendre qu'il aurait pu en dire beaucoup plus, mais qu'il choisit la tempérance, la conciliation » (Jaubert $2008: 115)^{3}$, on est en présence d'une figure argumentative : qui dit ethos, dit argumentation. L'ethos, ou l'image que le locuteur donne de lui-même à travers son discours, est une composante argumentative qui résulte de l'adaptation de ses propos au destinataire. Aussi n'est-il favorable que s'il est convaincant, i.e. lorsque l'image de l'argumentateur favorise la persuasion de l'argumentataire, contribue à le séduire. Cette entreprise peut avoir un résultat souhaité non seulement grâce à un euphémisme, mais aussi à son contraire : le dysphémisme ou l'hyperbole. Comme le remarque M. Bonhomme (2009 : 6-7), « la tension énonciative manifeste de l'hyperbole augmente la portée argumentative du discours. En se plaçant d'emblée au terminus d'une échelle évaluative, cette tension produit des "arguments exagérés" qui renforcent les croyances (Berrendonner, 1981) ». Les moyens argumentatifs sont en nombre illimité, chacun pouvant aider à mener à bien un projet de persuasion à sa propre façon, par des voies diverses.

Or l'exemple du Monde, présenté ci-dessus à titre introductif, n'a pas été choisi au hasard. Depuis plusieurs années, nos recherches se concentrent sur la manière dont l'argumentation peut être effectuée dans le discours à l'aide de noms propres. Nous croyons en effet qu'ils peuvent être pris en charge avec succès dans des situations de communication visant à persuader.

Dans cet article, nous nous intéresserons aux néologismes propriels et à leur fonction argumentative dans le discours. En prenant pour observatoire quelques innovations construites sur le sigle $D S K^{4}$, nous voudrions notamment (dé)mon-

\footnotetext{
${ }_{2}$ Le Monde (17.09.2011), <https://www.lemonde.fr/a-la-une/article/2011/09/17/la-vie-de-bureauapres-dsk_1573703_3208.html>.

${ }^{3}$ La linguiste le dit à propos de la litote, mais c'est vrai aussi pour l'euphémisme dont celle-là est, selon A. Jaubert, « une ramification particulière » (ibid.).
}

${ }^{4}$ Nous avons soumis à une analyse non exhaustive un corpus constitué d'énoncés glanés çà et là 
trer que leur emploi suppose une coprésence de plusieurs fonctions, en apparence centrées exclusivement sur le destinateur, mais qui sont là pour appuyer la fonction persuasive, donc axée sur le destinataire, de ces unités.

\section{Coprésence et hiérarchie des fonctions des néologismes propriels}

Comme le remarque Jakobson (1963:214),

« [...] il serait difficile de trouver des messages qui rempliraient seulement une seule fonction. La diversité des messages réside non dans le monopole de l'une ou l'autre fonction, mais dans les différences de hiérarchie entre celles-ci. La structure verbale d'un message dépend avant tout de la fonction prédominante. Mais [...] la participation secondaire des autres fonctions [...] doit être prise en considération par un linguiste attentif ».

Il s'en suit qu'un énoncé peut avoir plusieurs fonctions dans des dosages différents, mais, si elles sont toutes à prendre en compte, on peut néanmoins dégager celle qui prévaut. Dans le cas qui nous retient ici, nous posons qu'il convient de placer au sommet de la hiérarchie fonctionnelle la fonction de persuasion et que toutes les autres fonctions lui sont subordonnées.

Arcand et Bourbeau (1995) proposent une hiérarchie des fonctions des messages suivant le critère intentionnel : « [1]a fonction dominante, disent-ils, est celle qui répond à la question : "Dans quelle intention ce message a-t-il été transmis ?" et [...] les fonctions secondaires sont là pour l'appuyer» (1995:27). Dans cette optique, il faut faire la différence entre la « fonction-fin » (visant l'effet persuasif que le discours cherche à produire) et les « fonctions-moyens » qui sont activées ponctuellement dans les énoncés pour servir la fin poursuivie.

\section{Argumentation : pour une conception inclusive}

Il faut tout d'abord préciser ce que nous entendons par « argumentation », notion qui est fort différemment définie selon le point de vue adopté. Parmi les différentes approches, nous privilégions celle proposée par R. Amossy (2006), qui seule permet de rendre compte des processus mobilisant les noms propres à des fins de persuasion. Dans cette optique, sans nous engager dans des considérations détaillées sur les théories existantes ${ }^{5}$, nous nous bornerons à relever ici deux avantages principaux de la conception d'Amossy :

sur Internet, relevant aussi bien du discours journalistique que de la « parole citoyenne » (forums, blogs, commentaires aux articles).

5 On en trouvera un tour d'horizon complet p. ex. dans Amossy $(2006,2012)$ et Amossy \& Koren (2009). 
1) L'argumentation est envisagée par elle en termes de visée, mais aussi de dimension argumentative ;

2) Elle n'exclut pas de son champ les arguments bâtis sur le plan de l'affectivité.

Le premier avantage réside en fait dans une vision large de l'argumentation. La linguiste propose en effet de substituer à l'opposition problématique de l'argumentatif et du non-argumentatif la conception des degrés d'argumentativité (ibid., p. 36). Dans un texte récent, l'auteure de cette conception la qualifie d'« étendue » et d'" inclusive » et explique ce qui la distingue des autres approches (2018: 1-2) :

«En effet, la dimension argumentative marque l'écart qui sépare une conception restreinte et une conception large ou étendue de l'argumentation (il va de soi que restreinte n'a ici aucun sens péjoratif). [...] La conception restreinte entend limiter l'argumentation au déploiement d'un discours qui use d'arguments pour prouver le bien-fondé d'une thèse ; elle l'étudie dans sa singularité en le différenciant de tout ce qui n'en relève pas de façon stricte. Dans ce sens, elle est donc exclusive. La conception étendue est inclusive : elle englobe l'argumentation comprise au sens strict et la place au cœur de ses préoccupations ; mais elle la situe au centre d'un continuum qui comprend à l'une de ses extrémités la polémique comme confrontation violente de thèses antagonistes, et de l'autre une orientation des façons de penser et de voir, de questionner et de problématiser, qui ne s'effectue pas par la voie du raisonnement formel » (c'est nous qui soulignons).

L'approche d'Amossy permet d'inclure, dans l'analyse argumentative, les textes n'ayant pas pour objectif avoué de persuader, mais susceptibles d'orienter le regard, d'inciter à réfléchir sur un sujet. Les innovations lexicales proprielles suscitent l'intérêt et frappent l'esprit en s'inscrivant ainsi dans une stratégie de séduction. Ils argumentent à leur façon ce que les approches restreintes ne pourraient pas cerner, refusant même de s'y pencher. Par exemple, ce n'est sans doute pas la même chose de dire " l'amour au sens DSKien du terme " ${ }^{6}$ que de dire « le sexe ». Le décodage des proprionymes s'effectue au niveau langagier, mais surtout au niveau culturel, ce pour quoi leur emploi constitue une marque de connivence.

Le second avantage est de faire la part belle à l'affectivité dans le processus d'argumentation : la rationalité, que d'autres chercheurs considèrent LE fondement de l'argumentation suffisant pour la soutenir, doit nécessairement être, selon Amossy (2008), « complémentée par l'affectivité pour le bon succès de l'entreprise de persuasion $»^{7}$. Cette approche est donc capable d'expliquer comment

\footnotetext{
${ }^{6} \mathrm{http}: / /$ lecatalog.com/coco-de-mer-nous-explique-a-quoi-nous-pensons-toutes-les-six-secondes.html

${ }^{7} \mathrm{https} / / /$ books.openedition.org/pur/30428?lang=fr
} 
l'emploi de néologismes propriels, qui fonctionnent sur le mode pathémique, peut influencer l'autre, modifier sa façon de penser et de voir. Si « [1]es théories de l'argumentation restreinte se focalisent sur le logos, y voyant avant tout un partage de la raison effectué sur la base d'arguments logiquement valides » (Amossy, ibid.), l'approche inclusive réévalue le rôle du pathos, de l'émotion, dans l'échange argumentatif.

C'est dans ce cadre méthodologique que nous allons voir de plus près certaines fonctions des néologismes forgés à partir du sigle propriel $D S K$, lesquelles appuient la fonction argumentative.

\section{Fonctions des néologismes propriels}

Selon J.-F. Sablayrolles (2000), les fonctions des innovations lexicales peuvent être centrées soit sur le locuteur, soit sur l'interprétant, soit sur la langue, ou avoir des sources, des intentions et des effets hétérogènes. De notre perspective d'analyse, ce classement est globalement rattachable aux trois pôles de l'argumentation: l'ethos, le logos et le pathos, centrés respectivement sur l'orateur (l'argumentateur), le discours et l'auditoire (l'argumentataire). Or une difficulté se présente ici à l'analyste : la mixité de certains cas rend délicate l'entreprise de classification en termes de fonctions. Comme nous l'avons déjà remarqué cidessus, il est rare qu'un message assume une seule fonction. Nous dirions même qu'elles sont indissociables. Autant dire que l'emploi d'un néologisme propriel n'est pas à rattacher à un seul pôle argumentatif.

Pour des impératifs d'espace, dans ce qui suit, nous n'allons explorer plus en profondeur que le pôle de l'ethos. Nous espérons montrer à cette occasion que les fonctions qu'il est communément admis de rattacher au destinateur, concernent celui-ci tout autant que le destinataire. La volonté d'influencer l'autre n'est pas sans incidence sur l'analyse fonctionnelle qui, dès lors, devient problématique : l'examen en termes de fonctions du langage telles que déterminées par Jakobson (1963) se voit ainsi contesté dans son point concernant le prétendu centrage de chacune des fonctions sur un seul élément du schéma de communication.

\subsection{Fonction conniventielle}

La fonction conniventielle des néologismes propriels est-elle centrée sur l'énonciateur qui veut construire son ethos de connivence, c'est-à-dire montrer qu'il est bien désireux de se trouver "sur la même longueur d'onde » que son partenaire d'échange ? Ou vise-t-elle ce dernier avec qui l'argumentateur veut assurer un lien qui lui permetra de persuader plus facilement? S'il est possible de la ranger du côté de l'argumentateur, c'est parce que c'est lui qui met en œuvre les 
dispositifs lui permettant d'instaurer des relations de complicité, mais on le voit bien : la connivence est un jeu à deux.

J.-B. Grize (1996:5) remarque qu'« [a]rgumenter est [...] une activité discursive qui, en tant que telle, exige une participation active de ceux auxquels on s'adresse, réclame même de leur part une certaine connivence ». En effet, celle-ci est indispensable pour réussir l'entreprise de persuasion. Dans le cas des néologismes propriels, on a affaire à la connivence culturelle, i.e. le décodage du message s'effectue surtout au niveau culturel :

(1) On est toujours friand de nouvelles recrues féminines sur ce site c'est pas faux $\ddot{\sim}$ (ça fait un peu DSKesque cette phrase $)^{8}$.

Dans cet énoncé, l'emploi de l'adjectif néologique DSKesque est un clin d'œil complice : le locuteur sait que la destinataire de son post est capable de comprendre le vrai sens de son message sur la base d'un savoir partagé, d'une doxa où sont inscrits les noms propres notoires ${ }^{9}$ relevant de la langue-culture qui leur est commune. Il emploie les lexèmes friand et féminine, qui peuvent avoir ensemble des connotations sexuelles, et il admet en être conscient (ça fait un peu DSKesque). Or il ajoute en même temps un «mais » sous-entendu : "ça fait un peu DSKesque [vicieux], mais ce n'en est pas le cas. Mon unique intention est de dire que les femmes sont bienvenues sur ce site où les hommes constituent la majorité des utilisateurs ». Il veut en persuader sa destinataire en jouant sur le mode du « vous et moi savons que... », donc sur l'implicite qui « produit un sentiment d'appartenance communautaire : on se sent au chaud, entre soi, dans cette communauté de "ceux qui savent ce que je veux dire". La connivence ainsi produite inclut et exclut à la fois : elle instaure une ligne de partage entre "nous" (qui nous comprenons à demi-mot) et "les autres" (écartés de la compréhension) » (Krieg $1999: 32$ ).

\subsection{Fonction euphémique}

Il faut avoir un fonds culturel commun pour interpréter correctement les messages contenant des néologismes construits sur les noms propres inscrits dans la mémoire collective et la doxa. Ceci est particulièrement vrai dans le cas de la fonction euphémique dont un exemple a déjà été évoqué en liminaire. Considérons-en un autre, dans lequel un blogueur décrit, dans un de ses billets, la ville de Carthagène des Indes en Colombie :

\footnotetext{
${ }^{8}$ https://www.enigme-facile.fr/noel-gagnez-des-indices-2558/comment-page-3.

9 Voir à ce sujet la très stimulante étude de Schapira (2010) qui propose une « définition doxale » pour les noms propres notoires.
} 
(2) L'urbanisme, enfin ce qui en tient lieu, pourrait être qualifié par un seul mot : la pagaïe (dans un blog de bonne tenue, comme celui-ci, on n'utilise pas le terme DSKien de b... !). Aucune planification apparente n'a présidé à l'édification des maisons, immeubles et constructions en tous genres ${ }^{10}$.

L'énonciateur abrège le mot trivial bordel à l'initiale seule, suivie des points de suspension. Pour que le destinataire puisse immédiatement repérer le mot ainsi euphémisé, il ajoute qu'il s'agit d'un « terme DSKien », en tablant sur la capacité de son partenaire à détecter les connotations sexuelles du sigle DSK. Parler dans ce cas d'une « double euphémisation » ne semble pas outrancier : dans la formule atténuante le terme DSKien de b..., l'adjectif DSKien contourne les réalités tabouisées à l'aide de connotations.

Mais il y a plus : l'exemple (2) est particulièrement intéressant parce qu'il montre comment les fonctions euphémique et conniventielle vont de pair dans l'argumentation. L'énonciateur souligne lui-même qu'il s'agit d'un blog « de bonne tenue » et, bien qu'il ne le qualifie pas d'adoucissant, son dire s'apparente au « jugement d'euphémisation » compris comme « le fait de désigner explicitement une formulation comme un "euphémisme" (ou de la qualifier d'“euphémique" ou d"“euphémistique") » (Krieg-Planque 2004 : 60). De plus, cette chercheuse remarque judicieusement que le jugement d'euphémisation « joue à la fois sur l'image de modération de celui qui ne dit pas tout (ménageant les choses et les personnes) et sur la connivence que le locuteur établit en laissant deviner ce qu'il aurait pu dire » (ibid., p. 67) ${ }^{11}$.

\subsection{Fonction expressive}

L'argumentateur peut pourtant compter sur le succès de son projet autrement que par la seule construction d'un ethos de modération. Il peut ainsi, au contraire, rejeter toute tempérance au profit de l'expressivité, s'il la juge plus efficace et qu'elle correspond mieux à son intention de faire adhérer son partenaire d'échange à sa vision.

Jakobson définit la fonction expressive (ou « émotive») comme visant « à une expression directe de l'attitude du sujet à l'égard de ce dont il parle. Elle tend à donner l'impression d'une certaine émotion, vraie ou feinte [...]» (op . cit., p. 214). Déjà s'il est question de feindre une émotion, n'est-ce pas pour produire un effet sur quelqu'un, i.e. influencer le récepteur ? Dans cette perspective, nous allons certes entendre l'expressivité comme un phénomène consistant à mettre en

\footnotetext{
${ }^{10} \mathrm{http} / /$ latina2015.com/fr/2015/02/cartagena-de-indias/.

${ }^{11}$ Soit dit en passant, l'emploi dans l'énoncé analysé du mot familier pagaïe, témoigne aussi d'une « intention conniventielle » de son auteur, ou du moins de la recherche d'une familiarité avec le destinataire.
} 
avant la subjectivité de l'énonciateur, mais surtout comme l'intensité de l'effet produit sur le destinataire par le choix des moyens « expressifs », c-à-d., traduisant énergiquement et de manière suggestive une pensée. Dans ce cas-là, la fonction expressive est à rattacher au pôle du pathos en plus de celui de l'ethos. Considérons l'exemple suivant :

(3) Retenons [...] ce chiffre effrayant, époustouflant, brandi comme une injonction à un peu de décence : 75000 viols par an, en France : Aha, on fait moins les malins ! Le message est donc clair : que tous ceux qui s'amusent et font de l'esprit sur la femme de chambre DSKisée se rappellent qu'en France, chaque année, on viole 75000 femmes, bordel !12

La fonction expressive de cet énoncé est patente : les exclamations, les interjections et le caractère émotif du propos traduisent bel et bien l'attitude de son auteure envers le viol et les sentiments qu'elle éprouve. Mais elle veut aussi faire partager sa vision et ses émotions au public, donc argumenter sur le mode pathémique. DSKiser devient un synonyme expressif de violer, condensant un maximum de contenu dans un minimum de substance d'expression.

Comme le fait remarquer Marcellesi, « l'emploi par le locuteur d'un néologisme [...] ne semble pas innocent ; son utilisation traduit la recherche d'un effet, d'une action produite sur le destinataire [...] $»(1974: 98)$. Selon Sablayrolles, « [on] peut décocher un néologisme comme on décoche un coup » (1993: 65 cité par Vorger 2015 : 156). Les néologismes propriels semblent doublement argumentatifs grâce à la connivence culturelle qu'ils activent. C'est justement la culture partagée qui rend possible le déchiffrage de l'intention de l'auteur du post ci-dessous, lequel use du procédé argumentatif efficace qu'est la resémantisation des sigles :

(4) Le problème des Délinquants Sexuels Kroniques, oui on peut les appeler les DSK, c'est qu'ils ne s'arrêtent pas, ils ne s'arrêtent jamais. C'est pour cela qu'il faut les surveiller les dénoncer les condamner. Les pédophiles et les DSK sont des prédateurs, qui chassent leur proie en prenant autant de plaisir qu'en les consommant. [...] Les DSK et les pédophiles recommencent toujours ${ }^{13}$.

Par ce commentaire à l'article traitant de la pédophilie à Hollywood, l'énonciateur non seulement exprime son indignation envers les auteurs d'infractions sexuelles de tous types, mais encore a toutes les chances d'influencer la façon de voir de ses partenaires d'échange éventuels. Il met à profit le potentiel argumentatif du nom propre Dominique Strauss-Kahn, lequel est dû surtout à

\footnotetext{
12 https://culturalgangbang.blogspot.com/2011_05_01_archive.html.

${ }^{13} \mathrm{https} / / /$ www.agoravox.tv/tribune-libre/article/l-avantage-d-epouser-sa-belle-5316 1.
} 
l'appartenance de ce nom ayant un sens associatif communément partagé à la mémoire discursive. Le public sait très bien ce que suppose ce oui on peut les appeler les DSK. Plus encore, l'argumentateur se rend compte de l'effet pathémique que le sigle est capable de produire : le nom propre est ici employé en antonomase, un trope qui, comme toute figure, donne à penser et oriente la vision. Appeler quelqu'un DSK au lieu de délinquant sexuel peut avoir pour but de provoquer chez le destinataire une réaction plus vive.

Grâce à son énorme pouvoir évocateur, le sigle $D S K$ est apte à connoter toutes sortes de pratiques sexuelles sortant du commun, à dire beaucoup en peu de mots. Expressif et économique, ce « sigle fourre-tout sexuel » est la partie accrocheuse de l'énoncé, l'élément qui frappe l'esprit et permet d'attirer l'attention du destinataire, comme dans l'exemple suivant :

(5) Rassurez-vous Between, le nouveau réseau social spécial couple, est arrivé. Ne vous fourvoyez pas, il ne s'agit pas là d'un site consacré à l'échangisme et autre réjouissance DSKienne, mais bien d'une appli iPhone ou Android dédiée au couple. C'est sûr ça manquait ! ${ }^{14}$

La valeur argumentative de l'adjectif néologique consiste ici à jouer sur l'implicite : le destinataire qui comprend son sens a « le même plaisir du déchiffrage $[. .$.$] et le même plaisir de l'entre-soi que produit le sentiment de la devinette$ réussie » (Krieg 1999: 32).

\section{Conclusion}

À la lumière des exemples présentés, la néologie prenant pour base le sigle $D S K$ apparaît comme un procédé argumentatif efficace. Dans le cas des noms propres, c'est la référence à la culture partagée qui apparaît comme le recours premier de l'argumentateur, car ils font partie du bagage culturel, d'un réservoir commun dans lequel les locuteurs puisent pour établir un rapport de connivence essentielle à la persuasion. Bien évidemment, pour qu'un nom propre appartienne à ce stock commun, il doit être plus ou moins notoire. C'est ce que Ch. Le Bart exprime en ces mots : " [1]e nom échappe à son propriétaire lorsque ce dernier commence à exister auprès du grand public » $(2000: 128)$.

Que notre analyse commence par la fonction conniventielle, n'est pas un hasard. La connivence semble le soubassement de toute argumentation. Les néologismes construits à partir de noms propres fonctionnent sur le mode de l'implicite, donnant ainsi à l'argumentateur la possibilité de réussir son projet de persuasion. Comme l'affirme Herman (2008: 197), « Il y a [...] une forme d'effet de club entre personnes qui ont le privilège de se comprendre au-delà de ce qui est dit ».

\footnotetext{
${ }^{14} \mathrm{https} / / / w w w . e l a e e . c o m / 2011 / 12 / 29 / 12061$-between-le-2-0-en-toute-intimite.
} 
La fonction argumentative des néologismes propriels suppose toujours la coprésence d'autres fonctions qui peuvent paraître primordiales. Cependant, elles lui sont toutes subordonnées au niveau du discours qui, même s'il n'est pas ouvertement argumentatif, comporte une dimension argumentative inhérente. Comme l'affirme Amossy, « on parle toujours pour et en fonction de quelqu'un » (2006 : 41). Nous avons abordé la fonction euphémique, mais la fonction dysphémique peut servir, elle aussi, les besoins de l'argumentation discursive, le dysphémisme étant expressif et l'expressivité appuyant l'argumentation.

La vitalité et de la longévité des néologismes sont en général imprévisibles, ce qui est particulièrement évident pour les innovations proprielles, en l'occurrence celles bâties sur le sigle $D S K$, si fortement liées à la durée du référent et à ses éventuels « exploits », réels ou prétendus.

\section{Références bibliographiques}

Amossy \& Koren 2009 : R. Amossy \& R. Koren, " Rhétorique et argumentation : approches croisées ", Argumentation et Analyse du Discours, $\mathrm{n}^{\circ} 2$. https://journals. openedition.org/aad/561. 06/02/2019.

Amossy 2006 [2000] : R. Amossy, L'argumentation dans le discours. Paris : Armand Colin.

Amossy 2008 : R. Amossy, «Dimension rationnelle et dimension affective de l'ethos », in M. Rinn (dir.), Émotions et discours. L'usage des passions dans la langue, Rennes : PUR, 113-125, In : https://books.openedition.org/pur/30428?lang=fr. 09/02/2019.

Amossy 2012 : R. Amossy, «Faut-il intégrer l'argumentation dans l'analyse du discours? Problématiques et enjeux ", Argumentation et Analyse du Discours, $n^{\circ}$ 9, In : https://journals.openedition.org/aad/1346. 06/02/2019.

Amossy 2018 : R. Amossy, «Introduction : la dimension argumentative du discours enjeux théoriques et pratiques ", Argumentation et Analyse du Discours, $\mathrm{n}^{\circ} 20$, In : https://journals.openedition.org/aad/2560 $.06 / 02 / 2019$.

Arcand \& Bourbeau 1995 : R. Arcand \& N. Bourbeau, La communication efficace : de l'intention aux moyens d'expression, Anjou (Québec) : CEC.

Berrendonner 1981 : A. Berendonner, Éléments de pragmatique linguistique, Paris : Minuit.

Bonhomme 2009 : Bonhomme, « De l'argumentativité des figures de rhétorique », Argumentation et Analyse du Discours, $\mathrm{n}^{\circ}$ 2, https://journals.openedition.org/aad/495. $06 / 02 / 2019$

Grize 1996 : J.-B. Grize, Logique naturelle et communications, Paris : PUF.

Herman 2008 : T. Herman, « La connivence entre le journaliste et son lecteur. Un lieu d'échange entre sciences du langage et de la communication », in M. Burger (dir.), L'analyse linguistique des discours médiatiques, Québec : Éditions Nota bene, 183-206. 
Jakobson 1963 : R. Jakobson, Essais de linguistique générale, Paris : Minuit.

Jaubert 2008 : A. Jaubert, « Dire et plus ou moins dire. Analyse pragmatique de l'euphémisme et de la litote », Langue française, $\mathrm{n}^{\circ}$ 160, Paris : Armand Colin, 105-116.

Krieg 1999 : A. Krieg, «Vacance argumentative : l'usage de (sic) dans la presse d'extrême droite contemporaine ", Mots. Les langages du politique, $\mathrm{n}^{\circ}$ 58, Lyon : ENS Éditions, 11-34.

Krieg-Planque 2004 : A. Krieg-Planque, « Souligner l'euphémisme : opération savante ou acte d'engagement? Analyse du "jugement d'euphémisation" dans le discours politique », SEMEN, n 17, Besançon : Presses universitaires de Franche-Comté, $59-79$.

Le Bart 2000 : Ch. Le Bart, « Nommer les hommes politiques : identités prescrites, stratégiques, polémiques », Mots. Les langages du politique, $\mathrm{n}^{\circ} 63$, Lyon : ENS Éditions, 127-133.

Marcellesi 1974 : Ch. Marcellesi, « Néologie et fonctions du langage », Langages, n 36, Paris : Didier-Larousse, 95-102.

Sablayrolles 1993 : J.-F. Sablayrolles, « Fonctions des néologismes », in C. Cortès (dir.), Cahiers du CIEL, Paris : Université Paris 7 Denis Diderot, 53-94.

Sablayrolles 2000 : J.-F. Sablayrolles, La néologie en français contemporain, Paris : Honoré Champion.

Schapira 2010 : Ch. Schapira, « Une définition doxale : les noms propres potentiellement métaphoriques », Publifarum, $\mathrm{n}^{\circ}$ 11, http://publifarum.farum.it/ezine_articles. php?id=141. 18/02/2019.

Vorger 2015 : C. Vorger, « À la recherche de l'argot perdu. Le slam et ses néostyles », Folia Litteraria Romanica, n ${ }^{\circ}$ 10, Łódź : Université de Łódź, 147-161.

Sitographie

http://latina2015.com/fr/2015/02/cartagena-de-indias/. 02/11/2018.

http://lecatalog.com/coco-de-mer-nous-explique-a-quoi-nous-pensons-toutes-les-sixsecondes.html. 06/02/2019.

https://culturalgangbang.blogspot.com/2011_05_01_archive.html. 17/02/2019.

https://www.agoravox.tv/tribune-libre/article/l-avantage-d-epouser-sa-belle-5316 1. $17 / 02 / 2019$.

https://www.elaee.com/2011/12/29/12061-between-le-2-0-en-toute-intimite. 18/02/2019. https://www.enigme-facile.fr/noel-gagnez-des-indices-2558/comment-page-3. 07/02/2019.

https://www.lemonde.fr/a-la-une/article/2011/09/17/la-vie-de-bureau-apresdsk_1573703_3208.html. 06/02/2019. 


\section{Агњешка Коновска \\ Аргументативна функција неологизама насталих од властитих имена (на примеру сигле DSK)}

У раду испитујемо неологизме настале на основу властитих имена за потребе дискурзивне аргументације. Фокусирамо се на неколико иновативних конструкција насталих од сигле $D S K$, и настојимо да покажемо како је за њихову употребу потребно присуство више различитих функција (,функције-средства“) које се активирају како би оствариле функцију убеђивања („функција-циљ“). Изучавајући неколико примера у којима се јављају неологизми настали од властитог имена (faire son DSK, être un DSK, dskiser, dskien, dskesque), откривамо главне аргументативне механизме својствене властитим именима и неологизмима насталим од њих - ослањање на импицитна или прећутна знања, функционисање на патемичкој равни, прибегавање заједничким културним предзнањима — а који су неопходни за њихово разумевање.

Кључне речи : аргументација, неологија, властито име, прећутна знања, функције језика 